\title{
Lithography-free high aspect ratio submicron quartz columns by reactive ion etching
}

\author{
D. A. Zeze, ${ }^{\text {a) }}$ D. C. Cox, B. L. Weiss, and S. R. P. Silva \\ Advanced Technology Institute, School of Electronics and Physical Sciences, University of Surrey, \\ Guildford, GU2 7XH, United Kingdom
}

(Received 21 July 2003; accepted 17 December 2003)

\begin{abstract}
We describe lithography-free fabrication of sub-micron surface features on quartz substrates by the reactive ion etching (RIE) in a $\mathrm{CF}_{4} / \mathrm{Ar}$ atmosphere. These submicron glass columns are well defined, have a high aspect ratio, with the underlying substrate being very flat. The geometry of the fabricated surface columns is dependent on the RIE process parameters. The analysis of these glass columns shows that a differential etching process takes place. The optical characterization of these samples shows a significant absorption at visible wavelengths whereas the relative transmission is very high in the infrared range, suggesting that these samples could potentially be used for wavelength selection device applications. (C) 2004 American Institute of Physics.
\end{abstract}

[DOI: $10.1063 / 1.1647280]$

Reactive ion etching (RIE) of high-purity quartz samples has been studied for a range of applications in microelectronics, including optoelectronic hybrid integration. ${ }^{1}$ The ability to fabricate well-defined structures on the surface of the substrates, with near vertical sidewalls, a high aspect ratio, and a relatively low surface roughness, is a key factor for many applications, e.g., microelectromechanical systems, NEMS, and integrated optical devices. ${ }^{2}$ For a number of applications, monitoring the surface roughness to produce features with controlled geometry is a necessity. In this letter, we report the fabrication of submicron glass columns on the surface of fused quartz. These etched features are reproducible, although the process requires further study to enable accurate control of all parameters.

RIE of quartz samples was conducted using a combined radio frequency and microwave plasma system operating at $160 \mathrm{~W}(-380 \mathrm{~V}$ self-bias) and $600 \mathrm{~W}$, for the rf and microwave sources, respectively, and described previously. ${ }^{3}$ The etching was carried out at a typical rate of $\sim 30 \mathrm{~nm} / \mathrm{min}$, using electronic grade $\mathrm{CF}_{4} / \mathrm{Ar}$ plasma that contains $37 \%$ $\mathrm{CF}_{4}$. Several very fine (VF) grade fused quartz samples mounted onto the same stage were etched in runs of 30-60 min, using an aluminum contact mask onto which $0.5 \times 4 \mathrm{~mm}$ trenches are defined. The specimens processed were cleaned using acetone, methanol, isopropanol, and deionized water to remove nonvolatile debris from the surface. The etch depths and rms surface roughness were measured using a surface profilometer, with a resolution better than $1 \mathrm{~nm}$, giving depths of $2.8 \pm 0.2$ and $8.4 \pm 0.3 \mu \mathrm{m}$ for samples etched for 1.5 and $5 \mathrm{~h}$, respectively. The surface topography was investigated by scanning electron microscopy (SEM), while the chemical composition was determined by energy dispersive $\mathrm{x}$-ray analysis [(EDX) with a detection threshold of $0.2 \mathrm{wt} \%$ for all elements]. The reflectance was measured at $15^{\circ}$ and $45^{\circ}$ detector collection angles, arbitrarily chosen.

\footnotetext{
a) Author to whom correspondence should be addressed; present address: University of Durham, School of Engineering, Durham DH1 3LE, UK; electronic mail: d.a.zeze@durham.ac.uk
}

The surface features observed on fused quartz substrates after 5 and $1.5 \mathrm{~h}$ reactive ion etching are shown in Figs. 1(a) and 1(b) and are well defined with regular geometric shape. These micron sized columns, which we call "rods," can be fabricated on very flat and smooth fused quartz surfaces. The root-mean-square (rms) roughness of the surface between the rods was measured to be better than $\pm 5 \mathrm{~nm}$, which is much less than the $\pm 9 \mathrm{~nm}$ of the reference-unetched samples. The well-defined rods are only located in the regions neighboring the two rounded edges of the etched trenches and their characteristics are summarized in Table I. The samples etched for $1.5 \mathrm{~h}$ [Fig. 1(b)] show two truncated conelike structures of $1.4 \pm 0.2$ and $0.84 \pm 0.1 \mu \mathrm{m}$ height with shallow recesses on

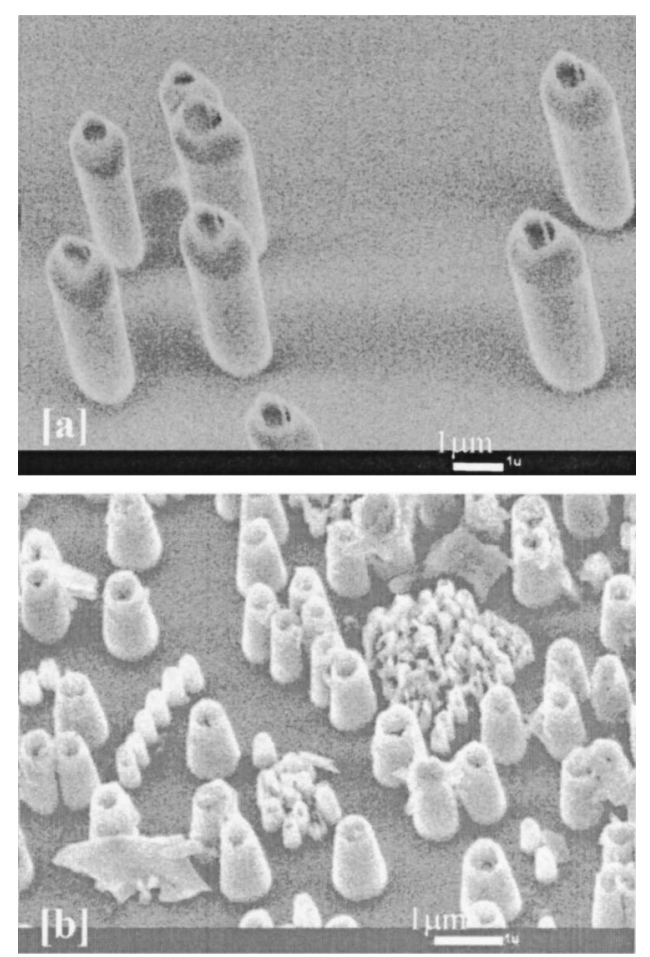

FIG. 1. The rods etched for $5 \mathrm{~h}$ are cylindrical and regular (a), while those etched for $1.5 \mathrm{~h}$ are truncated cones (b). 
TABLE I. Characteristics of the glass columns fabricated at the surface of the quartz substrate function of the etch time.

\begin{tabular}{cccccc}
\hline \hline $\begin{array}{c}\text { Time } \\
(\mathrm{h})\end{array}$ & $\begin{array}{c}\text { Etch depth } \\
(\mathrm{nm})\end{array}$ & $\begin{array}{c}\text { Rods height } \\
(\mathrm{nm})\end{array}$ & $\begin{array}{c}\text { Base } \\
\text { diameters } \\
(\mathrm{nm})\end{array}$ & $\begin{array}{c}\text { Density } \\
\text { rods }\left(\mathrm{cm}^{-2}\right)\end{array}$ & $\begin{array}{c}\text { Etch rate } \\
\text { of the rods } \\
(\mathrm{nm} / \mathrm{min})\end{array}$ \\
\hline 5.0 & 8400 & 3700 & 1800,1800 & $0.7 \times 10^{7}$ & 15.6 \\
1.5 & 2800 & 1400 & 1010,700 & $6.0 \times 10^{7}$ & 15.7 \\
1.5 & 2800 & 840 & 350,250 & $\cdots$ & 21.8 \\
19.0 & 45000 & 20000 & Variable & $\cdots$ & 17.5 \\
\hline \hline
\end{tabular}

the top of the rods which have diameters of $\sim 0.7$ or $\sim 0.25$ $\mu \mathrm{m}$, respectively. After $5 \mathrm{~h}$ of etching, the features become cylindrical rods $(3.7 \pm 0.2 \mu \mathrm{m}$ high and nearly identical) and the recess at the top surface is also seen in all features, Fig. 1(a). The density of these rodlike features, estimated at $\sim 6$ $\times 10^{7}$ and $\sim 0.7 \times 10^{7} \mathrm{~cm}^{-2}$ for 1.5 and $5 \mathrm{~h}$ etched samples, can be controlled by varying the elongation of the trenches and the process time. The average density of the specimens etched for $5 \mathrm{~h}$ (calculated taking a similar surface area for both sets of samples), is an order of magnitude lower than that of those etched for $1.5 \mathrm{~h}$ as smaller rods sitting between larger ones are etched away after $5 \mathrm{~h}$. The aspect ratio and the etch profile of the samples for various times were estimated using the evaluation software of the SEM system to determine the height, width, and taper angle of the rods. The taper angle was estimated to be $\sim 100^{\circ}$ and $90^{\circ}$ for samples etched for 1.5 and $5 \mathrm{~h}$, respectively. This corresponds to an aspect ratio better than 100:1, clearly indicating that nearcylindrical rods are produced. This work demonstrates that very high aspect ratio structures can be produced by RIE without resorting to photolithographic definition of the features, in contrast to Auciello et al. ${ }^{4}$ who produced silicon posts for field emission display applications using conventional photolithography and RIE. The rods described here were produced by monitoring carefully the etching process parameters, e.g., gas mixture, self-bias, process time, etch rate, given the structural properties of the fused quartz substrates. This etching produces a relatively flat surface in the middle of the trenches where no distinct rod structures are found and $\sim 40 \pm 10$ and $150 \pm 20 \mathrm{~nm}$ rms surface roughness were measured after 1.5 and $5 \mathrm{~h}$, respectively. Interestingly, the rms roughness of the middle of the trench scales linearly with time while the rms roughness of the surface between rods does not scale in this manner. The fact no significant $\mathrm{x}$-ray diffraction peak was observed under glancing angle analysis suggests that rods of various sizes may be separated by amorphous material. The presence of rods only in the rounded corners indicates a difference in the etch rate in this region (Table I).

The fused quartz trace element data provided by the manufacturer indicate that the $\mathrm{SiO}_{2}$ matrix primarily contains $\mathrm{Al}, \mathrm{Na}$, and $\mathrm{Ca}$, and differ from the experimental trace ele-

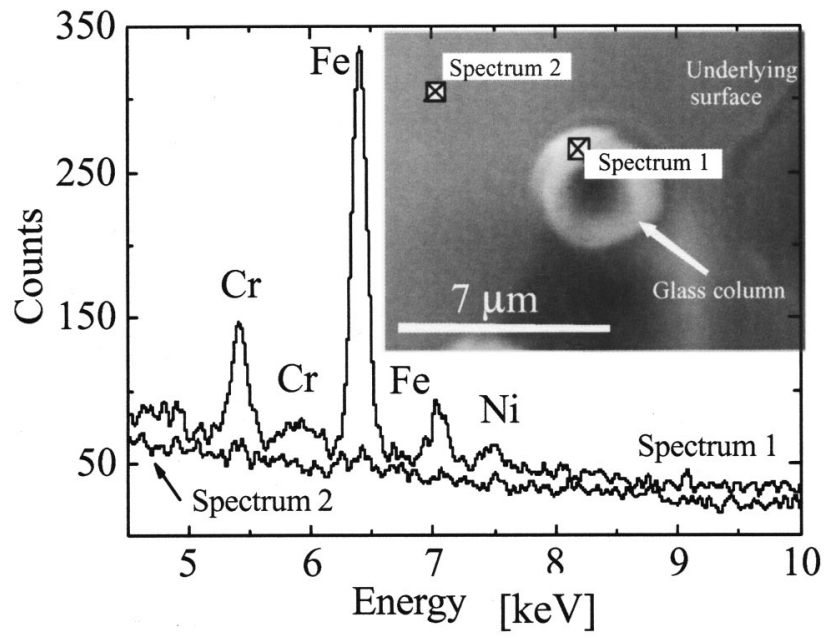

FIG. 2. Typical EDX spectra of a glass column and the underlying surface (inset SEM picture). The impurities are mostly localized to the rods (spectrum 1).

ments found here (Table II). The presence of these impurities may play a role in the formation of the rods since they can alter the basic quartz matrix and its properties. A nonuniform distribution of the impurities would induce a degree of difference in the selectivity and etch rates at local domains. These impurities include those produced by the etching process and the residues sputtered off the mask, e.g., fluorine, argon, carbon, and mask metal. Indeed, Fig. 2 compares the typical EDX spectra of the rods (spectrum 1) and that of the underlying surface (spectrum 2), shown on the inset SEM image. These spectra provide the evidence of the presence of the impurities and their nonuniform distribution across the substrate. Impurities, such as $\mathrm{Cr}(0.14$ at. \%), $\mathrm{Fe}(0.76$ at. \%), and $\mathrm{Ni}(0.05$ at. \%), are mostly concentrated in the rods while the underlying surface essentially contains $\mathrm{SiO}_{2}$. Note that $\mathrm{Al}, \mathrm{Mg}, \mathrm{F}$, etc., are also present in the rods at a lower-energy range. The presence of residual fluorine is a direct consequence of the etching process. However, metallic impurities can act as localized nanoscale masks when they aggregate randomly in the etched trenches, inducing local differences in selectivity. Therefore, the nonuniform distribution of the impurities across the substrate plays a major role in the differential etching.

The presence of well-defined rods observed in the rounded corners only, suggests a difference in the distribution of electrical field across the trench. The distribution of the electrical field around the edges of the trenches, was simulated using finite element analysis solving either Laplace's or Poisson's equations for metallic or dielectric substrates. $\Delta \phi=-\rho / \epsilon_{0}$, where $\phi$ is the electric potential, $\epsilon$ is the permittivity and $\rho$ is the charge density. The relative permittivity $\epsilon_{r}$ was set between 5.0 and 7.0. The enhancement factor $\beta$, that is the ratio between the local and the

TABLE II. The trace element composition of fused quartz VF grade provided by the manufacturer experimental EDX data (in at. \%) extracted from samples exhibiting $\sim 20 \times 4 \mu \mathrm{m}$ rods.

\begin{tabular}{lcccccccccccc}
\hline \hline \multicolumn{1}{c}{ Elements } & $\mathrm{Al}$ & $\mathrm{Ca}$ & $\mathrm{Cr}$ & $\mathrm{Cu}$ & $\mathrm{Fe}$ & $\mathrm{K}$ & $\mathrm{Li}$ & $\mathrm{Mg}$ & $\mathrm{Na}$ & $\mathrm{Ti}$ & $\mathrm{Ni}$ & $\mathrm{S}$ \\
\hline Manufacturer data (ppm) & 20.0 & 1.0 & 0.1 & 0.1 & 0.8 & 0.8 & 1.0 & 0.1 & 1.0 & 1.0 & $\cdots$ & $\cdots$ \\
EDX data (at. \%) & 0.18 & 0.10 & 0.14 & $\cdots$ & 0.68 & $\cdots$ & $\cdots$ & 0.13 & 0.26 & $\cdots$ & 0.05 & 0 \\
\hline
\end{tabular}




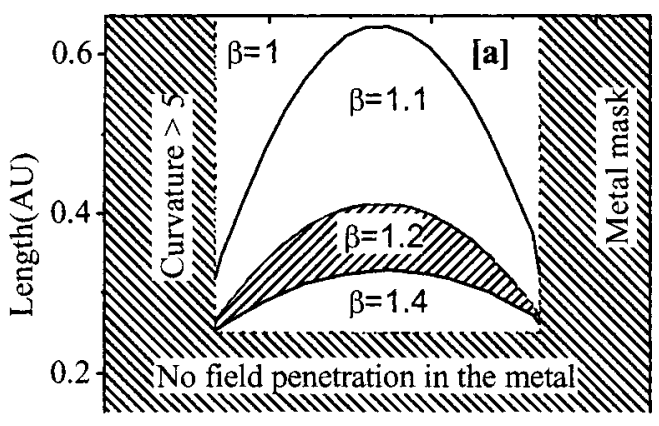

No field enhancement in the middle of the trench

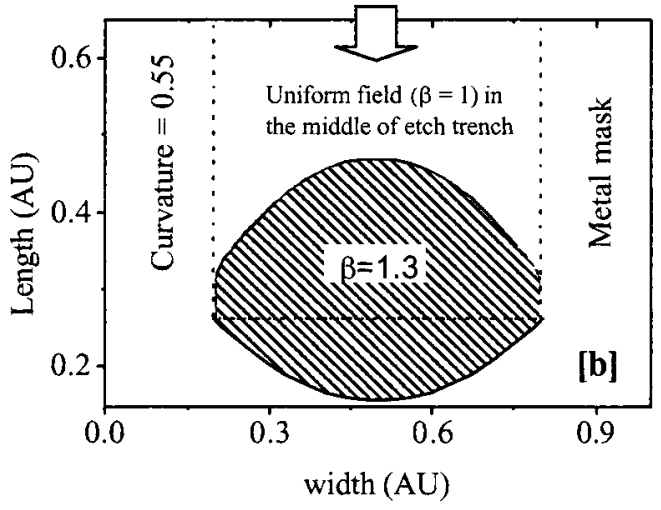

FIG. 3. The variation of electrical-field distribution in rounded corners of the etch trenches is illustrated for radius of curvature, $C \geqslant 5$ (a) and $C=0.55$ (b). The enhancement factor _ varies with $\mathrm{C}$. The field distribution is uniform in the middle of the trench in contrast to the rounded corners.

applied electric fields, is illustrated in Fig. 3 as a function of the radius of curvature $(C)$ at the end of the trench. $\beta$ greater than 1.0 is indicative of high-field concentrations, which is strong at the rounded corners (up to 1.4), uniform in the middle of the trenches and is dependent on the curvature (Fig. 3). Further away from the rounded corners, the field concentration decreases as three different $\beta$ values are observed for $C \geqslant 5$ [Fig. 3(a)] whereas only one zone is seen for $C=0.55$ [Fig. 3(b)]. $\beta$ greater than 1.0 is due to the differences in conductivity and the lower-field penetration in the metal mask. Recently, Macak et al. ${ }^{5}$ have also shown that the edge-related effects have a significant influence on the morphology, composition and thickness during deposition. Highfield enhancement at the edges affect the ion flux and angle of incidence during the process, therefore, one cannot discount the contribution of the edge effects in the localized differential etching that takes place. As a result, the combination of the electrical-field distribution and that of the metallic impurities leads to the formation of the glass columns.

The preliminary reflectance spectra normalized to the unetched sample are shown in Fig. 4. The relative reflectance is greater at infrared wavelengths from $800-1100 \mathrm{~nm}$. At $15^{\circ}$ collection angle (curves $\mathrm{c}$ and d), the relative reflectance is minimum around $550-560 \mathrm{~nm}$ while it is maximum around $990-1000 \mathrm{~nm}$. However, the intensity is dependent on the size of the rods. In turn, at $45^{\circ}$ (curves a, b), the maxima are further apart, with the minimum relative reflectance occurring at $\sim 430 \mathrm{~nm}$ and $\sim 540 \mathrm{~nm}$ for 4 and $20 \mu \mathrm{m}$ rods, respectively, while the maximum is $\sim 1100 \mathrm{~nm}$. The shift ob-

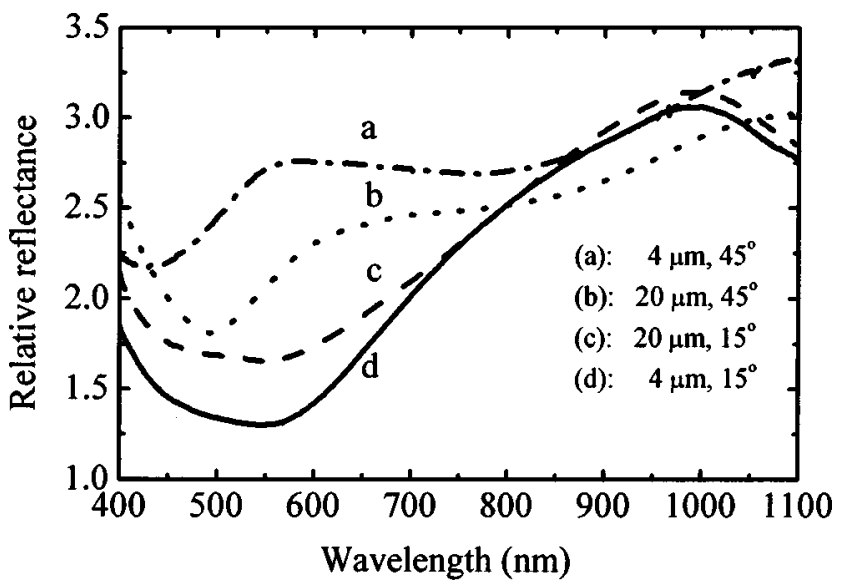

FIG. 4. Relative reflectance spectra normalized to unetched samples are shown for 4 and $20 \mu \mathrm{m}$ quartz columns at $15^{\circ}$ and $45^{\circ}$ collection angles.

served in the position of the peaks is indicative of the angular dependence. The spectra in Fig. 4 clearly show that the optical properties of the etched structures are dependent both on the angle of collection and the geometry of the rods present on the surface, as demonstrated previously ${ }^{6}$ for metallic diffraction gratings. The preliminary data above suggest that the rods could potentially be used as wavelength selection devices, providing the process is optimized to more accurately control the geometry, e.g., shape, size, and position of the rods at the surface of the substrates.

We have demonstrated a simple method of fabricating high aspect ratio glass rods on fused quartz, without using photolithography. It consists of carefully controlling the plasma parameters to fabricate high-density and high aspect ratio (better than 100:1) glass rods, which exhibit regular conical or cylindrical geometry. The differential etching leading to the formation of glass columns is attributed to the presence of localized metallic impurities in the quartz matrix and the nonuniform distribution of the electrostatic fields at the rounded edges of the contact mask. The preliminary optical data shows a significant absorption at visible wavelengths whereas the relative transmission and reflectance are dominant in the infrared, suggesting that these glass columns could be useful for many optical applications.

The authors acknowledge the financial support of the Engineering and Physical Sciences Research Council and the collaborative partners at the University of Kent and the Department of Chemistry at the University of Surrey. They also thank Jeff Hosea and Steve Greaves for the reflectivity and XRD measurements.

${ }^{1}$ C. Peirrat, T. Siegrist, J. De Marco, L. Harriot, and S. Vaida, J. Vac. Sci. Technol. A 14, 63 (1996).

${ }^{2}$ K. D. Skeldon, J. Mackintosh, M. von Gradowski, S. Thieux, and R. Lee, Pure Appl. Opt. 3, 183 (2001).

${ }^{3}$ D. A. Zeze, R. D. Forrest, J. D. Carey, D. C. Cox, I. D. Robertson, B. L. Weiss, and S. R. P. Silva, J. Appl. Phys. 92, 3624 (2002).

${ }^{4}$ O. Auciello, J. C. Tucek, A. R. Krauss, D. M. Gruen, N. Moldovan, and D. C. Mancini, J. Vac. Sci. Technol. B 19, 877 (2001).

${ }^{5}$ E. B. Macak, W. D. Munz, and J. M. Rodenburg, J. Appl. Phys. 94, 2829 (2003).

${ }^{6}$ R. A. Watts, T. W. Preist, and J. R. Sambles, Phys. Rev. Lett. 79, 3978 (1997). 